Artigo Original

\title{
Validação do Questionário de Ambiente de Grupo (GEQ) para a língua portuguesa
}

\author{
José Roberto Andrade do Nascimento Junior ${ }^{1}$ \\ Lenamar Fiorese Vieira ${ }^{1}$ \\ Antonio Fernando Boleto Rosado ${ }^{2}$ \\ Sidónio Serpa ${ }^{2}$ \\ ${ }^{1}$ Programa de Pós-Graduação Associado em Educação Física, \\ Universidade Estadual de Maringá/Universidade Estadual de Londrina, PR, Brasil \\ ${ }^{2}$ Faculdade de Motricidade Humana, Universidade Técnica de Lisboa, Portugal
}

\begin{abstract}
Resumo: O objetivo deste estudo foi traduzir o Group Environment Questionnaire (GEQ) para a língua portuguesa e verificar sua validade por meio da análise fatorial confirmatória, consistência interna e fidedignidade teste-reteste. Participaram do estudo sete profissionais (quatro tradutores e três professores doutores em Psicologia do Esporte) e 502 atletas de modalidades coletivas do estado do Paraná. A versão na língua portuguesa foi obtida por meio do método de tradução cross-cultural. Para a análise dos dados, utilizaram-se a análise fatorial confirmatória, o alfa de Cronbach e o coeficiente de correlação intraclasses. Os resultados evidenciaram que a versão para a língua portuguesa contém questões claras e pertinentes, apresentando o Coeficiente de Validade de Conteúdo (CVCt) > 0,70; apresenta consistência interna satisfatória $(r>0,70)$ e fidedignidade teste-reteste aceitável $(r>0,80)$. A análise fatorial confirmatória demonstrou que os itens 2 ("Eu estou feliz com a quantidade de tempo que tenho jogado") e 5 ("Alguns dos meus melhores amigos estão nesta equipe") não apresentaram confiabilidade individual aceitável, sendo assim excluídos. O modelo com 16 itens apresentou índices de ajustamento satisfatórios de validade fatorial confirmatória. Concluiu-se que a versão brasileira do GEQ demonstrou boas características psicométricas e se mostrou válida para avaliar a coesão de grupo em equipes esportivas.
\end{abstract}

Palavras-chave: Validação. Coesão de grupo. Esporte.

\section{Validation of the Group Environment Questionnaire (GEQ) for Portuguese language}

Abstract: This study aimed to translate the Group Environment Questionnaire (GEQ) to the Portuguese language and check its validity by validating the items through confirmatory factor analysis, internal consistency and test-retest reliability of this version. The subjects were seven professionals (four translators and three doctors in Sport Psychology) and 502 athletes of different kinds of collective sports of the state of Paraná. The Portuguese version was obtained from the method of cross-cultural translation. For data analysis it was used confirmatory factor analysis, Cronbach's alpha and the intraclass correlation coefficient. The results showed the Brazilian version contains clear and relevant items, showing Coefficient of Content Validity $(\mathrm{CVC})>0,70$; it shows satisfactory internal consistency $(r>0,70)$ and acceptable test-retest reliability $(r>0,80)$. The confirmatory factor analysis showed that items 2 ("I'm happy with the amount of playing time I get") and 5 ("Some of my best friends are on this team") don't demonstrated acceptable reliability individually, thus they were excluded; the model with 16 items had satisfactory indices of fit of confirmatory factorial validity. It was concluded that Brazilian version of the GEQ has shown good psychometric characteristics and it proved to be valid to assess the group cohesion in sports teams.

Keywords: Validation. Group cohesion. Sport.

\section{Introdução}

$\mathrm{Na}$ área de estudos da dinâmica de grupos na Psicologia do Esporte, a maioria dos trabalhos (AOYAGl et al., 2008; GOMES et al., 2008; CHIOCCHIO; ESSIEMBRE, 2009; BORREGO et al., 2010; TOROS, 2011) tem utilizado o Group Environment Questionnaire (GEQ), de Carron et al. (1985), um instrumento multidimensional que busca avaliar e entender o ambiente de grupo de equipes esportivas. No entanto, em um exame da literatura, não encontramos estudos que verifiquem a validade do GEQ no contexto brasileiro, o que demonstra a escassez de instrumentos que avaliem o ambiente de uma equipe esportiva. 
Atualmente uma das maiores dificuldades dos pesquisadores da área da Psicologia do Esporte no Brasil está relacionada à utilização de questionários, uma vez que o número de instrumentos validados para a língua portuguesa é reduzido. Para que um questionário seja utilizado como um instrumento psicológico em pesquisas, é fundamental que seja validado para a população na qual será realizada a investigação (VALLERAND, 1989).

No Brasil, Pasquali (2007) afirma que tem aumentado o número de estudos que se valem de instrumentos de avaliação psicológica em diferentes contextos, sendo o ambiente esportivo um dos cenários que vem ganhando grande destaque. Contudo, na maioria das vezes, os pesquisadores recorrem a instrumentos de outros países, sem demonstrar preocupação com a avaliação da qualidade de tais recursos (PASQUALI, 2007). Nesses casos, não se verificam a validade interna e a aplicabilidade para o contexto brasileiro (MARTINS, 2006). Considerado esse panorama, faz-se necessária a adaptação desses instrumentos estrangeiros para a realidade brasileira (PASQUALI, 2010). Essa transposição é possível por meio dos procedimentos de tradução cross-cultural, validação confirmatória, consistência interna e fidedignidade teste-reteste (VALLERAND, 1989; MAROCO, 2010).

O GEQ tem sido o foco de validação em países como Canadá (BRAWLEY et al., 1987), Portugal (BORREGO et al., 2010), Espanha (ITURBIDE et al., 2010), França (HEUZÉ; FONTAYNE, 2002), Nova Zelândia (LEESON; FLETCHER, 2005) e Estados Unidos (EYS et al., 2007). Esses estudos evidenciam que para países da Europa, América do Norte e Oceania, o GEQ apresentou indicadores adequados de validade, demonstrando a estabilidade do instrumento. Contudo, a literatura (MAROCO, 2010; PASQUALI, 2010) aponta que instrumentos avaliativos podem sofrer interferência em seus resultados quando aplicados a contextos socioeconômicos e culturais diferentes, evidenciando a importância da validação para a realizada ao qual será utilizado.

O GEQ foi elaborado por Carron et al. (1985) para a avaliação da coesão de grupo de equipes esportivas adultas, sendo validado com uma amostra de atletas adultos (a partir de 17 anos) de diferentes modalidades esportivas. Estudos apontam o GEQ como o melhor instrumento contemporâneo de medição para a coesão de grupo no esporte (CARRON et al., 2002; CARRON et al., 2005; GOMES et al., 2008). Recentemente, Eys et al. (2007) revisaram o GEQ com o objetivo de melhorar suas propriedades psicométricas, transformando todos os itens em afirmações positivas e concluíram que 0 questionário com todos os itens positivos teve uma consistência interna forte. Esse resultado sugere a utilização do instrumento revisado para a realização de pesquisas, uma vez que possui melhor consistência interna em relação ao questionário original.

No Brasil, esse instrumento revisado não tem sido utilizado por pesquisadores, uma vez que a tradução e a validação para o português ainda não foram realizadas. Ademais, os próprios autores do instrumento sugerem que, em uma perspectiva cross-cultural, os pesquisadores devem verificar se o conteúdo desse instrumento criado na cultura canadense é relevante no contexto em que será utilizado (EYS et al., 2007). Esta é uma lacuna metodológica na área da Psicologia do Esporte no Brasil e que o presente estudo pretende explorar com a validação do GEQ para a avaliação da coesão de grupo em equipes esportivas.

Diante disso, o presente estudo teve como objetivo validar para a língua portuguesa o Group Environment Questionnaire (GEQ), buscando especificamente avaliar a validade fatorial, a confiabilidade (consistência interna) e a fidedignidade teste-reteste do instrumento.

\section{Métodos}

\section{Participantes}

Participaram deste estudo sete profissionais (quatro tradutores juramentados e três doutores em Psicologia do Esporte), os quais aceitaram de forma livre e esclarecida desenvolver os processos de tradução, adaptação e validação de conteúdo do GEQ (CARRON et al., 1985). Um grupo de 20 atletas de ambos os sexos, selecionados por conveniência e estratificados por gênero, avaliou as questões do instrumento no que diz respeito à linguagem e à forma como o conteúdo estava sendo apresentado (validade aparente ou de face), de acordo com as recomendações de Maroco (2010).

Participaram da amostra de validação 502 atletas adultos do gênero masculino e feminino 
das regiões norte, noroeste e oeste do Paraná com idade média de 20,5 $\pm 3,5$ anos. O tamanho da amostra foi determinado com base nas recomendações de Pasquali (1999), que propõe no mínimo 10 participantes por item do instrumento. Dos atletas, 206 eram do sexo feminino e 296 do sexo masculino. Os esportistas estavam distribuídos entre as seguintes modalidades coletivas: futebol (97), futsal (83), voleibol (142), basquetebol (55), handebol (60), rúgbi (49) e vôlei de praia (16).

A seleção da amostra foi não probabilística por conveniência e os critérios de seleção das equipes foram os seguintes: 1) ter participado de alguma competição de nível nacional durante a temporada 2010; 2) ter participado do Campeonato Estadual da Modalidade durante a temporada 2009/2010; e 3) estar classificada para os Jogos Abertos do Paraná 2010, representando assim as equipes de alto rendimento do estado do Paraná. Além desses critérios, apenas os atletas que faziam parte da equipe há mais de três meses participaram da pesquisa, visto que esse pode ser considerado um período mínimo para o atleta ser capaz de analisar a coesão de grupo de sua equipe. Todos os atletas assinaram o Termo de Consentimento Livre e Esclarecido. A escolha por estas modalidades esportivas (futebol, futsal, voleibol, basquetebol, handebol, rúgbi e vôlei de praia) se deve a fato do instrumento analisar especificamente equipes esportivas coletivas.

\section{Instrumento de validação}

$O$ instrumento original utilizado foi o GEQ, revisado e modificado com itens apenas positivos (EYS et al., 2007). Tal questionário é composto por 18 itens que avaliam a coesão de grupo em equipes esportivas e se distribuem em quatro dimensões: 1) Integração no grupo-tarefa, 2) Integração no grupo-social, 3) Atração individual para o grupo-tarefa e 4) Atração individual para o grupo-social. As respostas são dadas em uma escala do tipo likert de nove pontos que variam de "discordo totalmente" (1) a "concordo totalmente" (9). Os resultados são atribuídos a cada subescala, obtidos com base na média aritmética das respostas dadas aos itens correspondentes a cada dimensão (CARRON et al., 1985) (Quadro 1).

Quadro 1. Dimensões do Group Environment Questionnaire GEQ.

\begin{tabular}{|c|l|c|}
\hline Dimensões & \multicolumn{1}{|c|}{ Descrição } & Itens \\
\hline $\begin{array}{c}\text { Integração no grupo-tarefa } \\
\text { (Gl-T) }\end{array}$ & $\begin{array}{l}\text { Sentimentos individuais dos membros em relação à } \\
\text { semelhança e à proximidade da equipe quanto às } \\
\text { tarefas a realizar. }\end{array}$ & $8,10,12,14,16$ \\
\hline $\begin{array}{c}\text { Integração no grupo-social } \\
\text { (Gl-S) }\end{array}$ & $\begin{array}{l}\text { Sentimentos e percepções individuais dos membros da } \\
\text { equipe quanto à semelhança e à proximidade no interior } \\
\text { da equipe como um todo, como unidade social. }\end{array}$ & $9,11,13,15$ \\
\hline $\begin{array}{c}\text { Atração individual para o } \\
\text { grupo-tarefa } \\
\text { (Al-T) }\end{array}$ & $\begin{array}{l}\text { Sentimentos individuais dos membros sobre seu } \\
\text { envolvimento pessoal com as tarefas, a produtividade, } \\
\text { as metas e os objetivos do grupo. }\end{array}$ & $3,4,6$ \\
\hline $\begin{array}{c}\text { Atração individual para o } \\
\text { grupo-social } \\
\text { (Al-S) }\end{array}$ & $\begin{array}{l}\text { Sentimentos individuais dos membros sobre seu } \\
\text { envolvimento pessoal, a aceitação e a interação social } \\
\text { com o grupo. }\end{array}$ & $1,2,5,7$ \\
\hline
\end{tabular}

Utilizou-se, também, a escala de clareza de linguagem e pertinência prática do tipo likert (em cinco pontos), a qual foi respondida pelos juízes avaliadores a fim de verificar a pertinência prática e a clareza de linguagem do instrumento traduzido (validade de conteúdo). Essas escalas permitiram investigar a consistência do julgamento das opiniões dos juízes avaliadores quanto aos aspectos relativos às questões do instrumento, partindo de "pouca pertinência /clareza" até "muita pertinência/clareza".

\section{Procedimentos}

Inicialmente, solicitou-se ao Professor PhD Albert Carron, autor do instrumento original no Canadá, a autorização formal para a tradução e a validação desse instrumento no Brasil. Tradutores juramentados e doutores na área da Psicologia do Esporte foram contatados para traduzir e avaliar a clareza e a pertinência dos itens do GEQ. O trabalho desse grupo de especialistas foi 
inicialmente individualizado e posteriormente realizado em conjunto.

Quanto à tradução dupla (reversa e independente - back translation), primeiramente dois tradutores de língua materna portuguesa, contratados de forma independente, traduziram para o português o GEQ; em um segundo momento, foram contratados dois outros tradutores, de língua materna inglesa, para converterem novamente para o inglês as versões traduzidas para o português. As duas traduções para a língua inglesa foram comparadas com a versão original do instrumento, a semântica das questões foi discutida e as mudanças necessárias foram realizadas. As versões na língua portuguesa foram revisadas e as correções dos termos técnicos utilizados na tradução e a adequação dos itens para a compreensão pelo público-alvo foram realizadas. As versões em língua portuguesa foram unificadas, resultando na versão final do instrumento (VALLERAND, 1989; PASQUALI, 2010). O instrumento traduzido para o português foi intitulado Questionário de Ambiente de Grupo (GEQ).

Quanto à participação dos juízes independentes, três professores universitários doutores na área de Psicologia do Esporte foram convidados a participar, no papel de juízes avaliadores, para verificar a adequação dos itens da versão original do GEQ. A esses profissionais coube responder e pontuar na escala likert - que parte de "pouquíssima pertinência/clareza" (1) e chega a "muitíssima pertinência/clareza" (5) - se, de acordo com sua experiência e seu conhecimento teórico, atletas adultos compreenderiam a estrutura de linguagem da questão (clareza) e se o item media o construto teórico que se propunha a avaliar (pertinência).

A análise teórica dos 18 itens iniciais do GEQ foi realizada por meio da técnica para avaliação da validade de conteúdo apresentada por Hernández-Nieto (2002). Essa técnica também verifica a concordância entre os juízes com relação à classificação dos itens nas dimensões.

A análise da validade de conteúdo, considerando a clareza da linguagem e a pertinência prática, foi realizada por meio do coeficiente de validade de conteúdo para cada item do instrumento $(\mathrm{CVCc})$ e para o questionário como um todo (CVCt). A validade de conteúdo da clareza de linguagem e da pertinência prática foi calculada com base nas respostas dos juízes.
Para tal, foi enviada aos três juízes uma planilha de avaliação da qual constavam os itens selecionados, três critérios para análise e um campo para observações, caso os juízes quisessem realizar sugestões, acrescentar ou retirar algum item. Os três critérios para análise foram a clareza de linguagem, a pertinência prática e a dimensão teórica à qual o item pertenceria.

Terminado $o$ processo de tradução e adaptação, foi realizado um estudo piloto com a versão final do instrumento, aplicado a 20 atletas de diferentes modalidades esportivas. Esse estudo possibilitou verificar o grau de entendimento dos atletas em relação aos itens do questionário traduzido e a compreensão de suas instruções e da utilização da escala de frequência. Não houve a necessidade de alteração no conteúdo do instrumento adaptado após a aplicação na amostra piloto. Posteriormente ao estudo piloto, foi programada a coleta de dados com os atletas participantes da amostra de validação.

Quanto à aplicação da versão traduzida do GEQ na amostra selecionada de atletas, foram realizados contatos com os diretores de esportes dos municípios das cidades das regiões norte, noroeste e oeste do Paraná, a fim de se obter autorização para a realização da pesquisa. $O$ projeto foi aprovado pelo Comitê Permanente de Ética em Pesquisa com Seres Humanos da Universidade Estadual de Maringá (Parecer $\mathrm{n}^{\circ}$ $648 / 2010$ ), e na sequência, foi enviado o termo de consentimento livre e esclarecido aos atletas das equipes que aceitaram participar da pesquisa.

A aplicação do GEQ foi realizada nos locais de treinamento dos atletas, em pequenos grupos antes dos treinamentos, com duração de aproximadamente 30 minutos. Com o fim de se obter os dados necessários à análise da fidedignidade teste-reteste, os atletas foram analisados em dois momentos distintos (inicial e retestagem) com intervalo de 7 a 14 dias após a testagem inicial.

\section{Análise dos dados}

Os dados foram avaliados com o auxílio dos softwares SPSS versão 19.0 e Amos versão 17.0. Para o cálculo do coeficiente de validade de conteúdo, utilizaram-se os princípios norteadores comumente aceitos na literatura especializada (HERNADEZ-NIETO, 2002), com ponto de corte 
de 0,70 . Os dados relativos à caracterização da amostra foram analisados de forma descritiva (média e desvio padrão) para dados contínuos e por distribuição de frequência (porcentual) para dados categóricos. Com o GEQ já vertido para a língua portuguesa, empregaram-se o coeficiente de confiabilidade alfa de Cronbach, a fidedignidade teste-reteste (coeficiente de correlação intraclasse) e a análise fatorial confirmatória para verificar a validade do instrumento, por meio dos índices de ajuste absoluto, parcimonioso e incremental recomendados por Maroco (2010).

\section{Resultados}

Análise teórica dos itens (validade de conteúdo do GEQ)

Verificou-se (Tabela 1) que todas as dimensões do GEQ obtiveram coeficientes de validade de conteúdo em relação à clareza de linguagem e à pertinência prática acima de 0,80 . Ao passo que o coeficiente de validade de conteúdo para o instrumento geral em relação à clareza da linguagem foi de 0.88 e para a pertinência prática foi de 0,92 . Assim, o coeficiente de validade de conteúdo geral (CVCt) do GEQ foi de 0,90 , indicando que o instrumento apresenta linguagem analisado.

Tabela 1. Coeficientes de validade de conteúdo para os critérios clareza de linguagem e pertinência prática na validação da versão brasileira do Group Environment Questionnaire (GEQ).

\begin{tabular}{lcc}
\hline \multirow{2}{*}{ Dimensões do QAG } & \multicolumn{2}{c}{ CVC $_{\mathbf{t}}$} \\
\cline { 2 - 3 } & Clareza & Pertinência \\
\hline Integração no grupo-tarefa & 0,95 & 0,96 \\
Integração no grupo-social & 0,88 & 0,96 \\
Atração individual para o grupo-tarefa & 0,93 & 0,95 \\
Atração individual para o grupo-social & 0,91 & 0,96 \\
Questionário total (QAG) & 0,88 & 0,92 \\
\hline
\end{tabular}

A classificação dos itens nas dimensões Integração no grupo-tarefa, Integração no gruposocial, Atração individual para o grupo-tarefa, Atração individual para o grupo-social foi avaliada pelo índice de concordância entre os juízes (coeficiente Kappa), que foi de 0,85. Além disso, foram discutidas em comitê (constituído por pesquisadores e juízes avaliadores) as observações e as sugestões dos juízes em relação à adequação dos itens do GEQ. Após o cálculo da validade de conteúdo e as adaptações dos itens, a versão final do instrumento foi concluída.

\section{Estatística descritiva e consistência interna do GEQ}

A análise descritiva dos resultados revelou que os atletas recorreram às nove possibilidades de resposta existentes para cada um dos 18 itens do GEG, com exceção dos itens 1 ("Eu aprecio fazer parte das atividades sociais desta equipe") e 10 ("Nossa equipe está unida na tentativa de alcançar seus objetivos de desempenho"). As médias das respostas situaram-se entre $5,77 \pm 2,28$ e 7,68 $\pm 1,67$ respectivamente nos itens 3 ("Eu sentirei falta dos membros desta equipe quando a temporada acabar") e 7 ("Eu gosto mais das festas da minha equipe mais que outros tipos de festas"). $O$ índice de consistência interna geral do GEQ foi de 0,81 . No entanto, como o GEQ é um instrumento multidimensional, torna-se mais importante calcular a confiabilidade de cada dimensão separadamente.

Verificou-se (Tabela 2) que o alfa de Cronbach das dimensões do GEQ variou de $\alpha=0,76$ a $\alpha=0,80$. Percebe-se, portanto, que o GEQ é um instrumento psicométrico confiável e de consistência interna satisfatória, visto que os valores de fidelidade de cada fator cumprem os critérios definidos por Hair et al. (2006), para os quais a consistência interna do fator deve ser igual ou superior a 0,70 (alfa de Cronbach: $\alpha$ $\geq 0,70$ ). 
Tabela 2. Consistência interna das dimensões da versão brasileira do Group Environment Questionnaire (GEQ) e correlação item-dimensão.

\begin{tabular}{cccc}
\hline Dimensões do QAG & $\boldsymbol{\alpha}$ & Item $\mathbf{n}^{\circ}$ & Correlação item-fator \\
\hline GI-T & 0,78 & $10,12,14,16,18$ & $0,81 / 0,73 / 0,80 / 0,80 / 0,71$ \\
GI-S & 0,80 & $11,13,15,17$ & $0,80 / 0,81 / 0,75 / 0,79$ \\
Al-T & 0,77 & $2,4,6,8$ & $0,68 / 0,77 / 0,70 / 0,79$ \\
Al-S & 0,76 & $1,3,5,7,9$ & $0,68 / 0,74 / 0,69 / 0,69 / 0,72$ \\
\hline
\end{tabular}

Nota: GI-T = Integração no grupo-tarefa; GI-S = Integração no grupo-social; Al-T = Atração individual para o grupotarefa; Al-S = Atração individual para o grupo-social.

Nota-se, também, a existência de correlações item-dimensão que variaram de $0,68 \leq r \leq 0,81$, indicando correlação moderada e forte entre as dimensões e seus respectivos itens. Os pesos fatoriais (as correlações entre os itens individuais e o fator) são considerados significativos quando o valor é igual ou superior a $0,5(P F \geq 0,50)$.

\section{Fidedignidade teste-reteste do GEQ}

Para análise da fidedignidade teste-reteste, calculou-se 0 coeficiente de correlação intraclasse. Verificou-se que, apesar da quantidade considerada elevada de pontos na escala intervalar proposta, a correlação encontrada pode ser considerada alta para um mesmo questionário psicológico aplicado duas vezes, com diferença de 7 a 15 dias entre os dois preenchimentos.

Os valores de correlação intraclasse apontaram a confirmação da fidedignidade (estabilidade) do instrumento. Todos os itens encontram-se acima do índice mínimo recomendado pela literatura $(r>0,80)$. Ao agrupar os 18 itens, encontrou-se correlação intraclasse média de 0,89 , evidenciando a fidedignidade dos itens do questionário. Por se tratar de um instrumento multidimensional, tornou-se imprescindível o cálculo do coeficiente de correlação intraclasse (teste-reteste) das quatro dimensões do GEQ.

Verificou-se que todas as dimensões do GEQ apresentaram coeficiente de correlação intraclasse entre $r=0,95$ e $r=0,96$, evidenciando uma forte fidedignidade entre teste e reteste para a amostra de validação. Confirmada a fidedignidade (estabilidade temporal) do questionário, a análise fatorial confirmatória do GEQ para a amostra analisada foi realizada a fim de saber se o modelo em quatro dimensões era suficiente para avaliar o constructo "coesão de grupo no esporte".

\section{Análise fatorial confirmatória do GEQ}

Antes de chegar aos cálculos relativos à validade fatorial confirmatória propriamente dita, uma análise preliminar foi efetuada a fim de constatar a adequação dos dados à análise. Trata-se precisamente da verificação da existência de casos aberrantes (outliers), visto que a inexistência desses casos é um pressuposto para esta análise. Os outliers foram avaliados por meio de medidas multivariadas, a distância de Mahanalobis. A verificação não evidenciou a existência de outliers, permitindo o uso da análise fatorial confirmatória. Observou-se, também, a normalidade, que é um dos pressupostos para a realização da análise fatorial confirmatória, tendo-se estudado não só a distribuição univariada dos dados (assimetria e achatamento), mas também a distribuição multivariada (coeficiente de Mardia para a curtose multivariada).

O modelo do GEQ submetido à análise fatorial confirmatória foi rigorosamente idêntico ao modelo de medida proposto pelos autores da versão original do instrumento, postulando a existência de quatro fatores (Integração no grupotarefa, Integração no grupo-social, Atração individual para o grupo-tarefa e Atração individual para 0 grupo-social). $O$ modelo proposto considera que cada item deve medir apenas um fator, diferenciando-se, assim, do modelo fatorial exploratório, em que cada item apresenta saturações fatoriais nos diversos fatores.

Avaliou-se a confiabilidade individual dos indicadores ou variáveis manifestas, procedimento particularmente apropriado para constatar a relevância dos indicadores nos modelos de medida. Com base nos resultados das regressões (coeficientes estandardizados), verificou-se que apenas os itens 2 ("Eu estou feliz com a quantidade de tempo que tenho jogado" - 
0,17 ) e 5 ("Alguns dos meus melhores amigos estão nesta equipe" - 0,45) não saturaram em seus respectivos fatores no sentido postulado. Todos os outros itens saturaram nos quatro fatores com uma magnitude superior a 0,50 $(p<0,001)$. Dessa forma, os itens 2 e 5 foram excluídos do modelo a ser testado pela análise fatorial confirmatória, visto que seus valores de confiabilidade individual foram inferiores a 0,50 (MAROCO, 2010).

Partindo-se da hipotética associação entre as dimensões do constructo avaliado para a amostra e seguindo-se os procedimentos de validação dos autores do instrumento original, a adequação do modelo fatorial confirmatório foi testada com o uso do método de estimação Maximum Likelihood (Máxima verossimilhança), que é o mais indicado em grandes amostras como a do presente estudo. Dessa forma, o modelo do GEQ foi testado por meio dos índices mais recomendados (MAROCO, 2010): X2 e p-value; X2/gl; CFI, GFI, TLI, NFI, AGFI, RMSEA (com I.C. 90\%) e p-value $(\mathrm{HO}: \mathrm{RMSEA} \leq 0,05)$. Esses índices têm como finalidade avaliar se o modelo apresenta um bom ajuste aos dados (Tabela 3).

Tabela 3. Índices de ajustamento da versão brasileira do Group Environment Questionnaire (GEQ) para a amostra de validação.

\begin{tabular}{cc}
\hline QAG & Valor \\
\hline$X^{2}$ & 309,72 \\
gl & 95 \\
p-valor & 0,000 \\
$X^{2}$ normalizado $\left(X^{2} / g l\right)$ & 3,02 \\
GFI & 0,93 \\
RMSEA & 0,06 \\
TLI & 0,92 \\
NFI & 0,91 \\
AGFI & 0,90 \\
CFI & 0,93
\end{tabular}

Nota. $X^{2}=$ Qui-quadrado; $\mathrm{Gl}=$ graus de liberdade; $\mathrm{X}^{2} / \mathrm{gl}=$ Qui-quadrado normalizado; GFI = Índice de qualidade do ajuste; RMSEA = Raiz do erro quadrático médio de aproximação; TLI = Índice Tucker-Lewis; NFI = Índice de ajuste normalizado; AGFI = Índice de qualidade de ajuste calibrado e CFI = Índice de ajuste comparativo.

Ao analisar a estrutura fatorial do modelo constituído por quatro fatores do GEQ, relativamente ao ajustamento global do modelo, verificou-se (Tabela 3 ) que a amostra avaliada apresentou um $X^{2}=309,72$ e significativo para $p<0,001$, sugerindo um fraco ajuste. No entanto, essa medida é muito sensível ao tamanho da amostra, composta por 502 atletas. Como são necessárias amostras volumosas para a realização da análise fatorial confirmatória, existe uma grande possibilidade de o qui-quadrado ser significativo mesmo quando o modelo apresenta um bom ajustamento aos dados. Apesar de ser comum utilizar e reportar os valores do teste $X^{2}$, outros índices são normalmente usados com mais confiança (BROWN, 2006), sendo "a estratégia mais defensiva para a avaliação dos modelos a consulta de índices de ajustamento de múltiplas classes" (HOYLE, 1995, p. 15). Portanto, é fundamental observar os outros índices que não são contaminados pelo tamanho da amostra.

As demais medidas de ajuste absoluto obtiveram valores aceitos pela literatura $(\mathrm{GFI}=0,93, \mathrm{RMSEA}=0,06)$. Todas as medidas de ajuste incremental (NFI=0,91, $\quad \mathrm{TLI}=0,92$, $A G F I=0,90)$ excederam o nível recomendado de 0,90, suportando, com credibilidade, a aceitação do modelo proposto. Quanto às medidas de ajuste parcimonioso, o qui-quadrado normalizado $\left(\mathrm{X}^{2} / \mathrm{gl}=3,02\right)$ foi adequado, considerados os níveis recomendados de 1,0 a 3,0 , e o CFI $(0,93)$ alcançou o nível recomendado $(>0,90)$. A Figura 1 apresenta as estimativas do modelo proposto, o qual descreve também a relação entre as dimensões e os indicadores na solução encontrada para os dados do GEQ. 


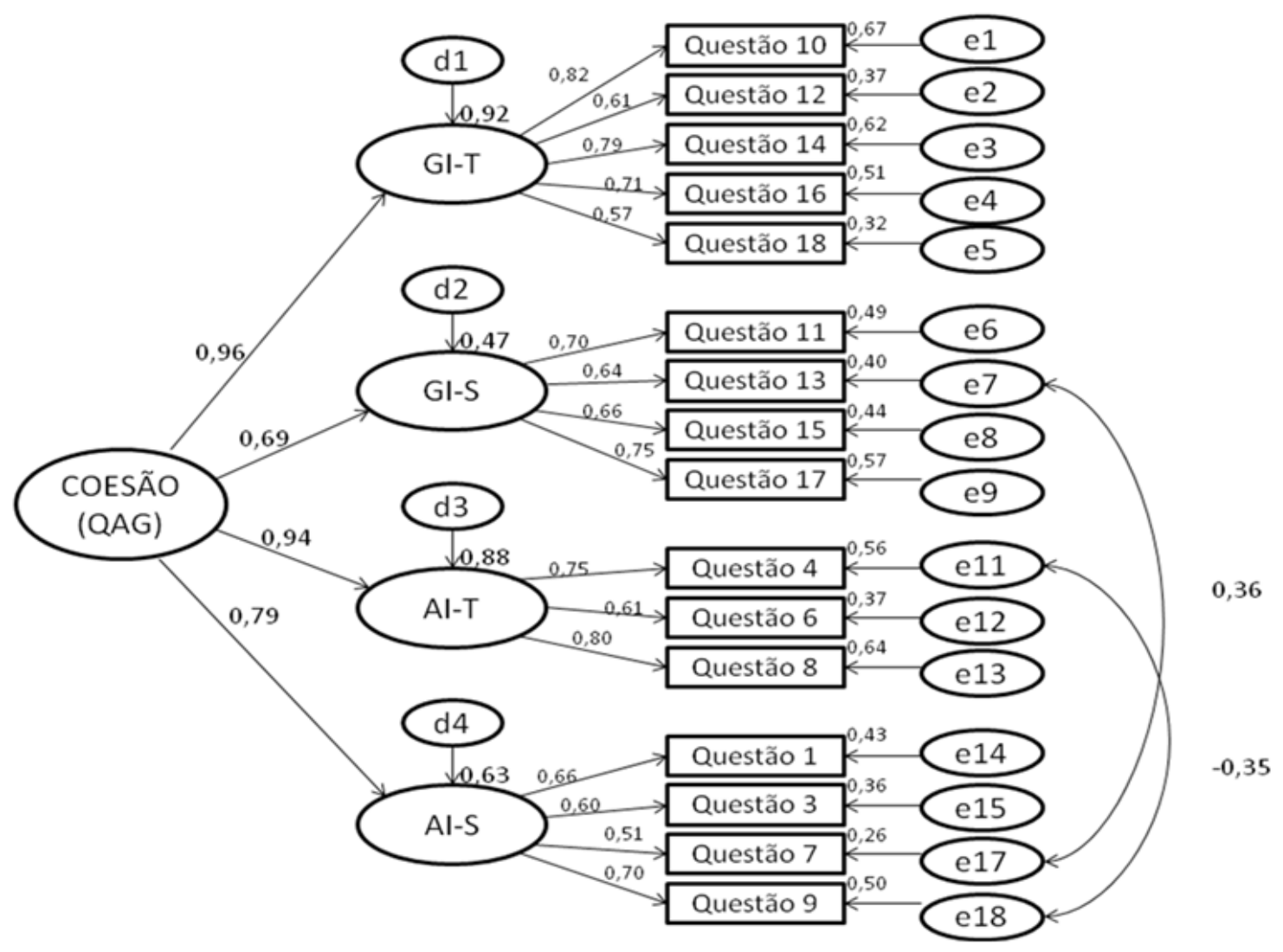

Figura 1. Carga fatorial, intercorrelações entre fatores e erro associado a cada item, no modelo de quatro fatores, com 16 itens da versão brasileira do Group Environment Questionnaire (GEQ).

$\mathrm{Na}$ análise da solução estandardizada expressa na Figura 1 (ou dos parâmetros estimados após a análise fatorial confirmatória), verificou-se que as saturações fatoriais $(\lambda)$ mostraram valores moderados situados entre 0,51 e 0,82 . Também os erros de medidas das variáveis observadas $(\delta)$ se revelaram moderados. Além disso, o modelo apresentou correlação entre os erros de alguns itens (7-“Eu gosto mais das festas da minha equipe mais que outros tipos de festas" e 17-"Os membros da nossa equipe mantêm-se juntos fora dos treinamentos e dos jogos"; 11-“Os membros da nossa equipe preferem sair para festas juntos do que sair sozinhos" e 18-"Nossa equipe se comunica abertamente sobre as responsabilidades de cada atleta durante as competições e torneios"), indicando a existência de itens problemáticos, que evidenciam alguma não ortogonalidade do modelo.

Para avaliar a validade convergente, empregou-se a análise da variância média extraída (VME), como sugerida por Fornell e
Larcker (1981), e valores superiores a 0,50 foram considerados indicadores de adequada validade convergente (HAIR et al., 2006). Os valores de VME foram os seguintes: $\mathrm{GI}-\mathrm{T}=0,67$; $\mathrm{GI}-\mathrm{S}=0,56$; $\mathrm{Al}-\mathrm{T}=0,52 ; \mathrm{Al}-\mathrm{S}=0,44$. Apenas este último fator apresentou uma validade convergente abaixo do limite proposto, entretanto, próxima do valor recomendado. $O$ fator de $2^{\mathrm{a}}$ ordem (coesão) apresentou uma variância média extraída de 0,72.

A solução encontrada no presente estudo é a mesma proposta no modelo original do GEQ, com exceção dos itens 2 ("Eu estou feliz com a quantidade de tempo que tenho jogado") e 5 ("Alguns dos meus melhores amigos estão nesta equipe"), que foram excluídos do modelo por não saturarem em seus devidos fatores com uma magnitude maior que 0,50 . Assim, a numeração e a reordenação dos 16 itens pelas quatro dimensões foram corrigidas para a finalização do processo de validação do GEQ, destacando que a estrutura da versão para a língua portuguesa do GEQ se alterou em relação ao modelo original de Eys et al. (2007). A versão brasileira do GEQ ficou 
com a seguinte distribuição: 1) Integração no grupo-tarefa (Itens 8, 10, 12, 14, 16); 2) Integração no grupo-social (Itens 9, 11, 13, 15); 3) Atração individual para o grupo-tarefa (Itens 3, 4, 6); e 4) Atração individual para o grupo-social (Itens 1, 2, 5, 7).

\section{Discussão}

O objetivo central do estudo foi determinar a confiabilidade, a fidedignidade teste-reteste e a validade fatorial do GEQ para a língua portuguesa como instrumento para avaliar a coesão de grupo em equipes esportivas e a adequação ao modelo conceitual de coesão de grupo desenvolvido por Carron et al. (1985).

Dessa forma, foram utilizados dados de um conjunto de atletas de sete modalidades coletivas diferentes (variando seu nível competitivo), com diferentes características dos membros (gênero, anos de experiência esportiva, tamanho da equipe e questões demográficas) em distintas fases de desenvolvimento do grupo, que compõem os participantes do presente estudo. De acordo com Carron et al. (1998), esse procedimento é fundamental para estabelecer condições mais aproximadas aos critérios sugeridos para testar o modelo conceitual de coesão em equipes esportivas.

\section{Estatística descritiva e consistência interna do GEQ}

$\mathrm{Na}$ estatística descritiva inicial, verificou-se que as variáveis observadas (18 itens) apresentaram uma distribuição normal. Resultados semelhantes foram encontrados em pesquisas internacionais de validação do GEQ ( HARMER, 1996; BORREGO et al., 2010; ITURBIDE et al., 2010).

Com a utilização do coeficiente alfa de Cronbach, tornou-se possível avaliar se todos os 18 itens do GEQ em suas quatro dimensões possuíam importâncias similares para o conjunto do questionário. $O$ valor de a varia entre 0,0 (mínimo) e 1,0 (máximo), sendo 0,70 os valores mínimos de aceitação recomendados (BLUNCH, 2008). A análise do grau de uniformidade ou de coerência existente entre as respostas dos atletas a cada um dos itens que compõem o GEQ (consistência interna) apresentou um índice de consistência interna geral de 0,81, evidenciando o aceitável e elevado alfa de Cronbach obtido pelo instrumento (NUNNALLY; BERSTEIN, 1994).
No entanto, como se trata de um instrumento multidimensional, mostrou-se mais importante calcular a confiabilidade de cada dimensão separadamente. Verificou-se que 0 alfa de Cronbach das dimensões do GEQ variou de $\alpha=0,76$ a $\alpha=0,80$, indicando uma forte consistência interna das dimensões. Logo, podese afirmar que os itens constitutivos das quatro dimensões do GEQ apresentaram precisão em suas medidas quando analisados em suas próprias dimensões.

Os coeficientes de consistência interna do presente estudo ( $\alpha=0,76$ a $\alpha=0,80)$ foram semelhantes à maioria das pesquisas internacionais de validação do GEQ (NTOUMANIS; AGGELONIDIS, 2004; LEESON; FLETCHER, 2005; BUTON et al., 2007; BORREGO et al., 2010; ITURBIDE et al., 2010), apresentando grande semelhança com os resultados de Eys et al. (2007), que revisaram e modificaram o GEQ para itens positivos, versão utilizada para a adaptação transcultural e validação para a realidade brasileira.

No entanto, não existe pesquisa de validação desse instrumento de avaliação da coesão de grupo no Brasil para que sejam feitas comparações com os resultados obtidos no presente estudo. Portanto, os resultados satisfatórios encontrados são fundamentais para a validação do GEQ no Brasil, visto que é o primeiro estudo de validação aplicado à realidade brasileira.

Além disso, os valores das correlações itemdimensão apresentaram-se bastante satisfatórios, variando de 0,68 $\leq r \leq 0,81$ e evidenciando que os itens realmente avaliam o que é proposto pelas dimensões, além de apresentarem correlação forte com suas respectivas dimensões (NUNNALLY; BERSTEIN, 1994). Correlações item-dimensão acima de 0,70 são consideradas indicativos de uma estrutura muito bem definida, pois o fator explica pelo menos $50 \%$ da variância do item (TABACHNICK; FIDELL, 1989; HAIR et al., 2006).

\section{Fidedignidade teste-reteste do GEQ}

Para muitos estudiosos, a fidedignidade está diretamente associada ao rigor e à precisão daquilo que se pretende medir, sendo o nível de fidedignidade de um instrumento fundamental para que ele seja válido (MEDEIROS, 1999; BALBINOTTI, 2005). Com a utilização do 
coeficiente de correlação intraclasse, tornou-se possível avaliar se todos os 18 itens do GEQ e suas quatro dimensões foram fidedignos após teste e reteste.

A análise evidenciou que tanto os 18 itens quanto as 4 dimensões do GEQ, não apresentaram adesão aos extremos, demonstrando a confiabilidade dos valores de correlação intraclasse observados. Em nenhum caso ocorreu o fenômeno da aquiescência (quer positiva ou negativa), o que indica tratar-se de dados confiáveis (NUNNALLY; BERSTEIN, 1994, BLUNCH, 2008). Cada item e dimensão do GEQ (teste) se mostrou satisfatoriamente correlacionado com seu próprio reteste e não foram observadas correlações negativas entre os itens $\mathrm{e}$ as dimensões (HERNANDEZ-NIETO, 2002).

Em relação à satisfatoriedade dos índices de coeficiente de correlação intraclasse teste-reteste, critérios mais flexíveis podem ser adotados, os quais aceitam valores acima de 0,70 . No entanto, se critérios mais rígidos são utilizados, os valores devem se situar entre 0,80 e 1,00 (NUNNALLY; BERSTEIN, 1994). Dessa forma, os resultados relativos à fidedignidade obtidos com os dados das duas aplicações do GEQ podem ser considerados satisfatórios, mesmo quando 0 critério adotado é o mais rígido. $O$ estudo original de validação do GEQ (CARRON et al., 1985) e a versão atualizada de Eys et al. (2007) não realizaram esta análise, impossibilitando a comparação dos resultados do presente estudo. Também não foram encontradas análises semelhantes nas demais pesquisas de validação do GEQ já realizadas internacionalmente.

\section{Análise fatorial confirmatória do GEQ}

De acordo com Pasquali (2010), a validação de constructo por meio da AFC é a forma mais fundamental de validade de um instrumento, uma vez que envolve a verificação científica da hipótese da legitimação da representação que os itens têm em relação ao modelo conceitual.

Nos dados analisados, constatou-se que a solução de 18 itens, com quatro fatores, não apresentava validade fatorial, uma vez que os parâmetros individuais dos itens 2 ("Eu estou feliz com a quantidade de tempo que tenho jogado") e 5 ("Alguns dos meus melhores amigos estão nesta equipe") não saturaram em seus respectivos fatores de acordo com os valores esperados de regressão (coeficientes estandardizados). Dessa forma, mantendo sempre como preocupação o respeito pela manutenção da integridade teórica do modelo, esses dois itens problemáticos foram eliminados do questionário, visto que seus valores de confiabilidade individual foram inferiores a 0,50, índice recomendado pela literatura (BALBINOTTI, 2005). Do mesmo modo, uma maior atenção deverá ser dada ao estudo da validade discriminante, averiguando se os diferentes fatores da coesão se distinguem significativamente entre si. A existência de correlações entre erros de diferentes fatores pode indicar uma fonte de variação comum dos itens não explicada pelos fatores comuns presentes no modelo ou uma não independência entre fatores, e essa hipótese exige confirmação em estudos futuros.

Outro aspecto problemático foi o qui-quadrado significativo ( $p<0,001)$, resultado que é tipicamente encontrado em grandes amostras (Tabela 3). Por essa razão, pesquisadores (COLE, 1987; MARSH et al., 1988) têm descartado esse dado de suas análises, visto que se trata de uma abordagem estatística extremamente sensível ao número de sujeitos da amostra (em amostras grandes esse resultado tende a ser sempre significativo). Por outro lado, as outras importantes medidas de adequação ao modelo multidimensional, o GFI $(0,93)$, RMSEA $(0,06)$, TLI $(0,92)$, NFI $(0,91)$, AGFI $(0,90)$, CFI $(0,93)$ e $X^{2} / g l(3,02)$ estão de acordo com os critérios de padrões recomendados pela literatura (FORNELL; LARCKER, 1981; BALBINOTTI, 2005), indicando um bom ajuste do modelo do presente estudo em relação ao questionário original.

Esses critérios múltiplos foram utilizados porque cada índice apresenta diferentes forças e fraquezas na avaliação da adequação do modelo fatorial confirmatório (BIDDLE et al., 2001). Podese notar que esses índices de ajuste do presente estudo estão de acordo com as expectativas, evidenciando a adequação geral do modelo em quatro fatores e a satisfatória validade fatorial confirmatória. Os índices de ajuste do modelo do presente estudo foram semelhantes ou melhores que a maioria dos estudos de validação do GEQ em diversos países do mundo (NTOUMANIS; AGGELONIDIS, 2004; LEESON; FLETCHER, 2005; BUTON et al., 2007; BORREGO et al., 2010; ITURBIDE et al., 2010), inclusive em relação à validação do instrumento original 
(CARRON et al., 1985), indicando bom ajuste do modelo para os atletas da amostra de validação e comprovando a satisfatória validade de constructo (fatorial) do instrumento para o contexto esportivo brasileiro. A revisão e modificação do GEQ por Eys et al. (2007), que foi a versão utilizada para a validação na presente pesquisa, realizou apenas a análise da consistência interna do instrumento, impossibilitando a comparação dos resultados, entretanto, os autores colocam este aspecto como uma limitação e sugerem a análise da validade fatorial do GEQ em futuros estudos, comprovando o avanço do presente estudo em relação à versão atualizada e revisada do GEQ por EYS et al. (2007).

Embora os resultados da análise fatorial confirmatória suportem uma validade convergente do modelo, verificou-se a existência de correlações entre os erros de alguns itens do modelo (Figura 1). Entretanto, essas correlações foram fracas e não significativas, não afetando, assim, a validade do modelo e sua adequabilidade. Estudos futuros devem reexaminar essas correlações entre erros e eventualmente reescrevê-los de forma a garantir uma estrutura fatorial mais clara. Segundo Blunch (2008), podem ser admitidas correlações entre erros se isso não colocar em causa a identificação do modelo, como é o caso do presente estudo.

Dessa forma, os resultados da validação do GEQ, em termos gerais, permitiram evidenciar boas qualidades psicométricas e afirmar que 0 GEQ (ANEXO) se adequa ao modelo de Carron et al. (1985). No entanto, é imprescindível salientar que o questionário foi reduzido a 16 itens, respeitando os critérios de parcimônia recomendados pela literatura (BROWN, 2006; BLUNCH, 2008), com uma nova ordenação e distribuição dos itens nas respectivas dimensões.

\section{Conclusão}

A versão para a língua portuguesa do GEQ apresentou resultados satisfatórios nas análises de conteúdo, confiabilidade, fidedignidade testereteste e validade fatorial confirmatória, evidenciando que a avaliação da coesão de grupo no contexto do esporte por meio do GEQ é válida e confiável. No entanto, é importante destacar que o questionário foi reduzido para 16 itens, respeitando os critérios de confiabilidade individual. Esses resultados apontam que 0 instrumento em análise realmente avalia a coesão de grupo por meio de quatro dimensões: integração no grupo-tarefa, integração no gruposocial, atração individual para o grupo-tarefa e atração individual para o grupo-social.

Com isso, os resultados obtidos por meio da versão brasileira do GEQ podem fornecer informações relevantes que auxiliem o técnico no desenvolvimento e na manutenção de um ambiente de grupo favorável dentro de uma equipe esportiva. Contudo, o estudo apresenta algumas limitações, visto que os atletas avaliados foram todos de equipes esportivas do estado do Paraná, estando limitado a amostras com características semelhantes à deste estudo. No entanto, estas equipes participam de competições de nível estadual e nacional e são constituídas por atletas de diferentes regiões do Brasil, indicando a representatividade da amostra. Estudos futuros devem dar corpo a essa preocupação de validação cruzada, de modo a avaliar se os resultados obtidos nesta amostra podem ser generalizados para outras amostras independentes. A replicação deste estudo com outras amostras de modo a confirmar a estabilidade da solução fatorial encontrada deverá ser testada. Do mesmo modo, o estudo da validade relacionada com o critério deverá ser explorado, examinando sua qualidade como preditor de comportamentos esportivos relevantes.

\section{Referências}

AOYAGI, M. W.; COX, R. H.; MCGUIRE, R. T. Organizational citizenship behavior in sport: relationships with leadership, team cohesion, and athlete satisfaction. Journal of Applied Sport Psychology, London, v.20, n.1, 25-34, 2008.

BALBINOTTI, M. A. A. Para se avaliar o que se espera: reflexões acerca da validade dos testes psicológicos. Aletheia, Canoas, v.1, n. 21, p.4352, 2005.

BIDDLE, S.; MARKLAND, D.; GILBOURNE, D.; CHATZISARANTIS, N.; SPARKES, A. Research methods in sport and exercise psychology: Quantitative and qualitative issues. Journal of Sports Sciences, London, v.19, p.777-809, 2001.

\section{BLUNCH, N. Introduction to Structural} Equation Modelling using SPSS and AMOS. London: Sage Publications, 2008.

BORREGO, C. C.; LEITÃO, J. C.; SILVA, C.; ALVES, J.; PALMI, J. Análise factorial confirmatória do group environment questionnaire 
com atletas portugueses. Avaliação Psicológica, Porto Alegre, v.9, n.3, p.359-369, 2010.

BRAWLEY, L. R.; CARRON, A. V.; WIDMEYER, W. N. Assessing the cohesion of teams: Validity of the Group Environment Questionnaire. Journal of Sport Psychology. Hyattsville, v.9, p.275-294, 1987.

BROWN, T. Confirmatory Factor Analysis for Applied Research. New York: The Guiford Press, 2006.

BUTON, F.; FONTAYNE, P.; HEUZÉ, J. P.; BOSSELUT, G.; RAIMBAULT, N. The QAG-A: an analog version of the questionnaire sur l'ambiance du groupe for measuring the dynamic nature of group cohesion. Small Group Research, Thousand Oaks, v.38, n.2, p.235-264, 2007.

CARRON, A. V.; BRAWLEY, L. R.; WIDMEYER, W. N. The measurement of cohesiveness in sport groups. In J. L. Duda (Ed.). Advances in sport and exercise psychology measurement. Fitness Information Technology, Inc. Morgatown. USA, 1998, p.213-226.

CARRON, A. V.; HAUSENBLAS, H. A.; EYS, M. A. Group Dynamics in Sport. Morgantown, WV: Fitness Information Technology; 2005.

CARRON, A. V.; WIDMEYER, W. N.; BRAWLEY, L. R. The Development of an instrument to assess cohesion in sport teams: The Group Environment Questionnaire. Journal of Sport Psychology. Hyattsville, v. 7, p.244-266, 1985.

CHIOCCHIO, F.; ESSIEMBRE, H. Cohesion and performance: a meta-analytic review of disparities between project teams, production teams, and service teams. Small Group Research, Thousand Oaks, v.40, p.382-420, 2009.

COLE, D. Utility of confirmatory factor analysis in test validation research. Journal of Consulting and Clinical Psychology, Washington, v.55, p.584-94, 1987.

EYS, M. A.; CARRON, A. V.; BRAY, S. R.; BRAWLEY, L. R. Item wording and internal consistency of a measure of cohesion: The Group Environment Questionnaire. Journal of Sport \& Exercise Psychology, London, v.29, p.395-402, 2007.

FORNELL, C.; LARCKER, D. F. Evaluating Structural Equation Models with Unobservable Variables and Measurement Error. Journal of Marketing Research, Chicago, v.18, p.39-50, 1981.

GOMES, A. R.; PEREIRA, A. P.; PINHEIRO, A. R. Liderança, coesão e satisfação em equipas desportivas: um estudo com atletas portugueses de futebol e futsal. Psicologia Reflexão e Crítica, Porto Alegre, v.21, n.3, p.482-491, 2008.

HAIR, J.; BLACK, W.; BABIN, B.; ANDERSON, R.; TATHAM, R. Multivariate Data Analysis. New Jersey: Pearson Educational, 2006.

HERNÁNDEZ-NIETO, R. A. Contributions to Statistical Analysis. Mérida: Universidad de Los Andes, 2002.

HEUZÉ, JP, FONTAYNE P. Questionnaire sur l'Ambiance du Groupe: A Frenchlanguage instrument for the measurement of group cohesion. Journal of Sport \& Exercise Psychology, London, v.24, p.42-67, 2002.

HOYLE, R. The Structural Equation Modeling Approach. Basic Concepts and Fundamental Issues. In R. Hoyle (Ed.). Structural Equation Modeling Approach: Concepts, Issues, and Applications. Thousand Oaks: Sage Publications, 1995,p.1-15.

ITURBIDE, L. M.; ELOSUA, P.; YANES, F. Medida de la cohesión en equipos deportivos. Adaptación al español del Group Environment Questionnaire (GEQ). Psicothema, Oviedo, v.22, n.3, p.482-488, 2010.

LEESON, H.; FLETCHER, R. B. Longitudinal Stability of the Group Environment Questionnaire With Elite Female Athletes. Group Dynamics: Theory, Research, and Practice, v.9, n.3, p.147160, 2005.

LI, F.; HARMER, P. Confirmatory factor analysis of the group environment questionnaire with an intercollegiate sample. Journal of Sport \& Exercise Psychology, London, v.18, p.49-63, 1996.

MAROCO, J. Análise de Equações Estruturais: Fundamentos teóricos, Software e Aplicações. Pêro Pinheiro: Report Number, 2010.

MARSH, H.; BALLA, J.; MCDONALD, R. Goodness-of-fit indexes in confirmatory factor analysis: The effect of sample size.

Psychological Bulletin, Washington, v.103,p.391-410, 1988.

MARTINS, G. A. Sobre confiabilidade e validade. Revista Brasileira de Gestão de Negócios, São Paulo, v.20, n.8, p.1-12, 2006.

MEDEIROS, E. B. Medidas psico \& lógicas: introdução à psicometria. Rio de Janeiro: Ediouro, 1999.

NTOUMANIS, N.; AGGELONIDIS, Y. A psychometric evaluation of the Group 
Environment Questionnaire in a sample of elite and regional level Greek volleyball players.

European Physical Education Review,

Thousand Oaks, v.10, n.3, p.261-278, 2004.

NUNNALLY, J. C.; BERSTEIN, I. H.

Psychometric theory. New York: McGraw-Hill, 1994.

PASQUALI, L. Instrumentos psicológicos:

Manual prático de elaboração. Brasília: LabPAM, 1999.

PASQUALI, L. Validade dos Testes Psicológicos: Será Possível Reencontrar o Caminho?

Psicologia: Teoria e Pesquisa, Brasília, v. 23, n. especial, p.99-107, 2007.

PASQUALI, L. Instrumentação psicológica: fundamentos e práticas. Porto Alegre, Artmed, 2010.

SCHUTZ, R. W.; EOM, H. J.; SMOLL, F. L.;

SMITH, R. E. Examination of the factorial validity of the Group Environment Questionnaire.

Research Quaterly for Exercise and Sport, Reston, v.65, n.3, 226-236, 1994.

TABACHNICK, B.; FIDELL, L. Using Multivariate Statistics. New York: HarperCollins Publishers, 1989.

TOROS, T. Analysis the differentiation between perceived coaching behaviors, goal orientation, team cohesion, perceived motivational climate and collective efficacy among the young male basketball players before and after the tournament. Pamukkale Journal of Sport Sciences. Denizli, v.2, n.1, p.27-39, 2011.

VALLERAND, R. J. Vers une méthodologie de validation transculturelle de questionnaires psychologiques: implications pour la recherche en langue française. Canadian Psychology, Winnipeg, v.30, n.4, p.662-671, 1989.

\section{Endereço:}

José Roberto de Andrade do Nascimento Junior Rua Tietê, 614/05 Jardim Universitário Maringá PR Brasil 87020-210

Telefone: (44) 9144-8849

e-mail: junior_jrs001@hotmail.com

Recebido em: 26 de abril de 2012.

Aceito em: 10 de dezembro de 2012.

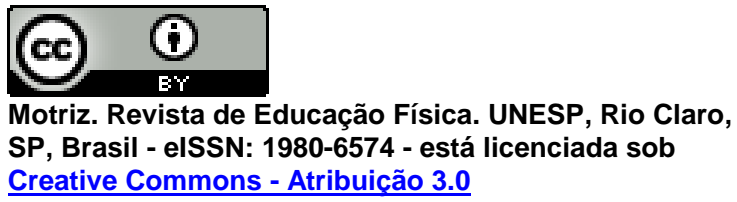

